we painted with the same rubber solution the outside of the plaster after the plaster had been applied, so as to make the cloth waterproof by the application of a thin coat of rubber. Incidentally, it may be stated that the same rubber solution with ether has been applied to the skin in order to prepare it for operation, instead of tincture of iodin, with very satisfactory results.

The reason adhesive plaster adheres better if the skin is painted with a solution of rubber is that the solution of rubber and ether adheres strongly to the skin, and naturally the adhesive plaster will in its turn adhere firmly to the rubber that covers the skin.

47 East Fifty-Seventh Street.

\section{A METHOD OF MOUNTING ANATOMIC AND PATHOLOGIC SPECIMENS IN GELATIN}

Kelley Haie, M.D., Wilmington, Ohio

The method here described is one by means of which specimens may be mounted between glass plates of any desired thickness or any size, depending on the character of the specimens.

1. The plates of glass are separated by strips of glass of any desired thickness. The strips should be one-half inch wide for ordinary work. For small or larger specimens, narrower or wider strips should be used.

2. Strips with square ends are cut the desired length by employing a piece of glass, the width of which is exactly equal to the length of the strips desired. An ordinary glass cutter and a draftsman's right angle rule or triangle are all that are required.

3. The strips are broken by holding the piece of glass in the left hand with the cut line down; then with a few swift, sharp strokes of the reverse end of the glass cutter, the glass opposite the cut line is fractured, and the strip is easily removed. As many strips as are needed may be cut.

4. The strips are then attached to the margins of the glass plate by means of Canada balsam from which the volatile principles have been driven off by heat. Optical houses can provide this material in stick form. A burning set employed by storage battery men is very effective in attaching the glass strips. A strip should be left out at one end.

5. The specimen or specimens are then attached to the plate by means of warm gelatin, in any position desired. They are kept in a horizontal position until the gelatin sets; then the plate with the specimens is immersed in liquor formaldehydi in order to render the gelatin insoluble and thus prevent the slipping of the specimen when mounted in warm gelatin.

6. Next the exposed side of the specimens is covered by attaching a glass plate to the glass frame with the volatilized Canada balsam.

7. After this the mounting process is finished by setting the glass case, with its contained specimens, in warm water. Liquid gelatin of any desired consistency is poured by means of a funnel into the glass case through the end that has been left open. The warm water prevents the gelatin's setting until all of the spaces have been filled and the air bubbles forced to the top of the gelatin. If the colors of the specimens have been preserved by Kaiserling's method, a jelly is prepared by adding gelatin, ten parts by weight to the third solution in which the specimens ordinarily are kept: water, 900 c.c.; glycerin, 540 c.c.; sodium acetate, $270 \mathrm{gm}$. A 1 per cent. solution of phenol (carbolic acid) is used for a preservative.

The gelatin should stop at a line corresponding to the inner edge of the glass strip that will be sealed in the end after the gelatin has solidified, and the exposed portion rendered insoluble by immersion in liquor formaldehydi. With care the glass case can be rendered air tight by closing the end with a strip of glass and the Canada balsam (stick).

8. Specimens can be labeled in a very simple manner. After the specimen is attached to the glass as described, the glass cover is placed in accurate position. With pen and drawing ink, ruled lines are drawn to parts that are indicated in printed letters. The cover glass is then removed and the inside thoroughly cleansed and the ruled lines and names traced through the glass with water-proof ink. Fine lines can be made by using black silkworm gut attached to the inside by means of thick gelatin. The lines and names are much plainer after they have been transferred to the inside of the cover glass. This is true, too, of the specimens when viewed through glass. They are mounted as described before.

\section{ADVANTAGES}

1. This method does away with large containers, such as bottles and jars, for small specimens.

2. It is very difficult to see a specimen in a bottle, and it is impossible to study it without taking it out, which will often lead to destruction. With this method the specimen is immobilized between two glass plates, is plainly visible and can be carefully studied without danger of destruction.

3. This rendering delicate specimens, such as embryos and biologic material, indestructible makes this method a great time saver to the instructor who wishes to use such material for teaching purposes, as he can use this material year after year without fear of having it destroyed.

4. Preservation of cross-sections of embryos, developmental stages of fishes, tadpoles, sections of brain, injected, etc., can be had with the utmost satisfaction.

5. Specimens can be filed away classified like lantern slides.

6. By using plate glass, even sections of the adult human being can be preserved.

7. Specimens preserved in Kaiserling's solution can be mounted in this way and kept without losing their color

8. This method can be used for displaying commercial products as well.

9. The specimens can be projected on the screen like any opaque objects, thus making it possible to use material at scientific meetings that could not be used otherwise.

\section{New and Nonofficial Remedies}

The following additional articles have BeEN ACCEPTED as conforming to the rules of the Council on Pharmacy and Chemistry of the American Medical Association for admission to New and Nonofficial Remedies. A copy of THE RULES ON WHICH THE COUNCIL bASES ITS ACTION WILL BE SENT ON APPLICATION.

W. A. Puckner, Secretary.

CULTURE-LAC.-A culture of Bacillus bulgaricus in whey marketed in bottles containing about 4 fluidounces.

Actions and Uses.-Culture-Lac is adapted both for internal and external use. See general article, Lactic Acid-Producing Organisms and Preparations (New and Nonofficial Remedies, 1919, p. 155).

Dosage.-From 4 to $8 \mathrm{Cc}$. (from 1 to 2 fluidrachms) in sweetened water one-half hour before meals. For infants 4 Cc. (1 fluidrachm) every two or three hours as directed by the physician. The date of issue is stated on each bottle Manufactured by the Geck Laboratory, New York. No U. S. patent.
U. S. trademark applied for.

Maggots in the Ear.-A full blooded Indian, aged 21, came to my office one evening, stating that on the previous afternoon a fly lit on his cheek, that he brushed it away but knocked it into his ear, and it crawled into the auditory canal. He tried to dislodge it but was unable to do so, although he thinks it flew out again in a few minutes. At 3 o'clock the next morning he was awakened by a severe earache, and later his wife got fourteen maggots out of his ear. On examination I saw moving about-and easily extractedsome maggots with small forceps. I then washed out the ear with boric acid solution and obtained at least seventy-five more maggots. They were about $2 \mathrm{~mm}$. in length, and quite thin. The patient had no further trouble.-Otro H. G. Rosfinkranz, M.D., Hoopa, Calif. 